\title{
Semantics for Connectivity Management in IoT Sensing
}

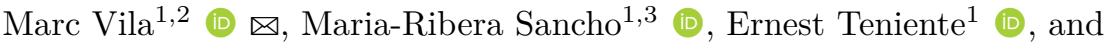 \\ Xavier Vilajosana ${ }^{2,4}$ (ID) \\ 1 Universitat Politècnica de Catalunya, Barcelona, Spain \\ \{marc.vila.gomez, maria.ribera.sancho, ernest.teniente\}@upc.edu \\ 2 Worldsensing, Barcelona, Spain \\ \{mvila, xvilajosana\}@worldsensing.com \\ 3 Barcelona Supercomputing Center, Barcelona, Spain \\ maria.ribera@bsc.es \\ 4 Universitat Oberta de Catalunya, Barcelona, Spain \\ xvilajosana@uoc.edu
}

\begin{abstract}
There are a large number of Internet of Things (IoT) devices that transmit information over the Internet, each with a different data format to denote the same semantic concept. This often leads to data incompatibilities and makes it difficult to extract the knowledge underlying that data. The only way to close this gap is to establish a common vocabulary in order to achieve interoperability between different sources and semantic data integration. This is the main goal of our proposal: to specify a general semantics for IoT sensing that allows the management of data between gateways, nodes, and sensors in a homogeneous way. Our proposal builds upon the joint Semantic Sensor Network and Sensor-Observation-Sample-Actuator (SSN/SOSA) ontology.
\end{abstract}

Keywords: IoT · Interoperability · Sensors · Ontologies · Semantics

\section{Introduction}

Kevin Ashton coined the term Internet of Things (IoT) about twenty years ago when he spoke of Radio Frequency IDentification (RFID) [11]. A Thing, in this context, is a physical object that aims to connect and exchange information with other devices over the Internet. Currently, there are more than 8 billion IoT devices communicating data periodically, heterogeneously, and globally.

The IoT world has evolved drastically since then, due to the need for comprehensive monitoring of different devices to provide real-time information about the status of a given domain.

Today, all IoT devices have the ability to communicate, at least, with their own ecosystems. What happens, though, when you need to include different devices from different manufacturers in the same system? All devices have their own properties, data format, and communication technologies. This makes it difficult to share information with each other.

To be able to provide generic solutions that apply to different monitoring environments and domains, we need to abstract from the particular syntax and 
data formats of the different devices and provide common semantics to all the data managed. We achieve it through the definition of an ontology for connectivity management in the IoT sensing domain. Ontologies provide several benefits: (1) share a common understanding of the structure of information among software agents; (2) enable the reuse of domain knowledge; (3) domain assumptions are made explicit; (4) domain and operational knowledge are kept separated [14].

In our understanding, an ontology is an abstract interpretation of the concepts and properties of the domain and of the data stored in the information base, in the same way as a data model is. Therefore, both ontology and data model can be understood as synonyms.

Our work is related to industrial research at Worldsensing cused on providing services through the monitoring of industrial environments from IoT devices. The development of a platform able to control and act on its own and third-party industrial IoT devices has become a critical goal for the success of the company, and the need to consider different types of devices can only be successfully addressed through this common vocabulary.

The ontology we propose aims to interact and manage a custom platform based on ThingsBoard 0 , an open source IoT platform for device management, data collection, processing and visualization. Our project will extend the management and interaction capabilities of ThingsBoard, not only to IoT devices but also to its network elements; sensors, nodes and gateways needed to communicate information over the Internet. Our ontology is specified as an instantiation of common sensing ontologies such as Semantic Sensor Network Ontology (SOSA/SSN) and the information gathered from their surrounding standards.

LoRat is the main technology used to communicate nodes and gateways. In [13], the authors compare three standards for transmitting low-power messages over a long distance; NB-IoT, Sigfox, and LoRa; and concludes that LoRa is currently the best option for smart monitoring applications. This technology makes it possible to operate low-cost devices with very long range, infrequent communication rates, and high battery longevity. However, it is beyond the scope of this paper to decide which technology will be used. Data packets have a maximum size of 243 bytes, sent over a configured schedule in a given installation. The advertised commercial maximum range is currently between 5 and $15 \mathrm{~km}[1]$.

Summarizing, we propose in this paper the Connectivity Management Tool Semantics (CMTS) ontology; an extension of the World Wide Web (W3C) and the Open Geospatial Consortium (OGC) joint Semantic Sensor Network and Sensor-Observation-Sample-Actuator (SSN/SOSA) ontology [10,12]. Our proposal makes use of two auxiliary ontologies for location (GeoSPARQL - Geographic Query Language for RDF Data) and temporal properties (OWL-Time Ontology). Our main contribution is to provide such a system, with the set of language elements needed to achieve semantics interoperability.

\footnotetext{
${ }^{5}$ Worldsensing: https://www.worldsensing.com

6 ThingsBoard: https://thingsboard.io

${ }^{7}$ Long Range - LoRa Alliance Standardisation Committee: https://lora-alliance.org
} 
We build upon and extend our previous work in [15] where we proposed a prototype to monitor IoT devices to automatically react and notify through alarms when data surpasses a certain threshold.

\section{Related Work}

\subsection{Semantic Web Background}

The World Wide Web uses the term "Semantic Web" to refer to the representation of the world data and vocabularies, with the objective of making it readable for humans and machines. In the Semantic Web, the information is written in the form of Triples, three entities with which to refer to a concept.

Consider the example geo:Barcelona geo:partOf geo:Catalonia, which follows the subject-predicate-object format. In this example; geo:partOf means that the geo 8 "Barcelona", is a location which is part of "Catalonia". Where the non-literal values are URIs9; like "Barcelona" or "Catalonia".

As mentioned in [3]; "the Resource Description Framework (RDF) is the basis of the Semantic Web. Things in the world are referred to as resources and they can be anything that someone might want to talk about. i.e. 'the value of $X$ ' or 'all the cows in Texas'". RDF is intended to be used for representing information about those resources in a graphical form.

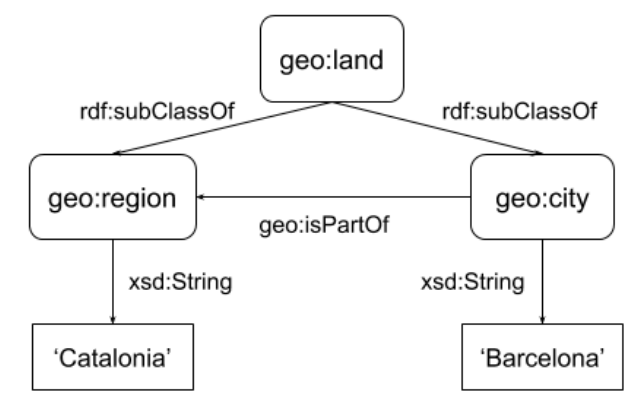

Fig. 1. Sample RDF in graphical notation

As shown in Figure 1; classes are rounded rectangles while literal values are represented in rectangles. The text linking classes refers to properties of an object, while the text linking classes and literal values refers to the property of the data to be displayed. The property rdf:subClass Of makes one class a subclass of another. Hence Region and City are both subclasses of Land. Whilst xsd stands for XML Schema Definition and indicates that the element linked is a String. So to say, "Catalonia" is an instance of Region but also of Land. At the same time, "Barcelona" is an instance of City but also of Land. And also that "Barcelona" is part of the "Catalonia" Region.

${ }^{8}$ Namespace made-up but understood to refer to geography.

${ }^{9}$ URI: Uniform Resource Identifier, a sequence of characters that identifies a resource. 


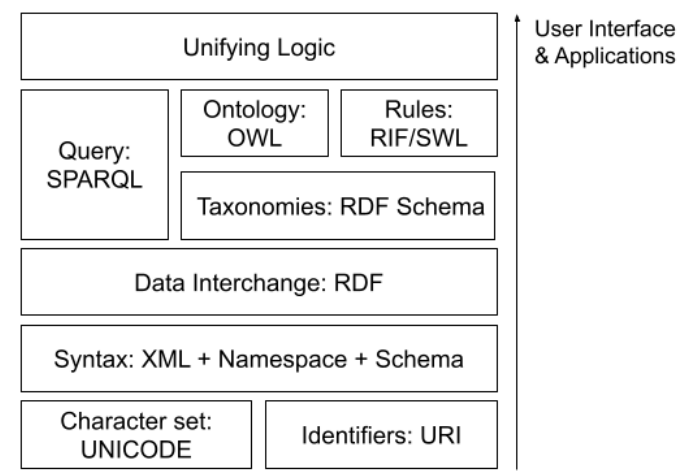

Fig. 2. Simplification of The Semantic Layer Web

Figure 2 represents a simplified view of the technological elements used in the "Semantic Web Architecture". Starting with the character set; UNICODE ensures that the language can be read by humans and computers using a standardized form. The resulting character string forms a URI. In the next layer, there is the Extensible Markup Language (XML), together with its Namespace and Schema, ensuring that the syntax used is common through the Semantic Web. Following this is the RDF we have just seen. Then, the RDF Schema (RDFS) provides guidelines on how to use or combine different RDFs and allows describing taxonomies of classes and properties to be used in lightweight ontologies. Going up, we find the OWL ontology; a language derived from logical descriptions that enable extending vocabulary over RDFS. At this point, RIF/SWL rules can be introduced in order to constrain the ontologies. Finally, the SPARQL -Simple Protocol and RDF Query Language- allow executing queries in RDF and SQL.

\subsection{Ontologies and Semantics in the Internet of Things}

The first ontology proposed for wireless sensor networks was defined in OWLLite 10 and dates back to 2004. In 2007, Eid et al. [7] proposed a universal ontology to solve the data heterogeneity problem in sensor networks.

The Open Geospatial Consortium (OGC) then published a series of standards with the aim of improving the interoperability of sensor and actuator systems. Among those that stand out in the subject treated are: Observations and Measurements $(\mathrm{O} \& M)^{11}$, which defines standard XML Schema and models for observations and features involved in sensor measurements; Sensor Model Language (SensorML)12, which provides robust and semantic means of defining characteristics and capabilities of sensors, actuators, and computational mechanisms; and Sensor Observation Service (SOS) 13 , which defines a web service for querying and manipulating observations and sensors.

10 OWL-Lite: https://www.w3.org/TR/owl-features/

11 Observations and Measurements (O\&M): https://www.ogc.org/standards/om

${ }^{12}$ Sensor Model Language (SensorML): https://www.ogc.org/standards/sensorml

13 Sensor Observation Service (SOS): https://www.ogc.org/standards/sos 
In 2011, the Semantic Sensor Network Incubator Group (SSN-XG) developed an ontology to describe sensors and sensor network resources [17]. They also gave recommendations for using the ontology to semantically enable applications and to provide better support for abstraction, categorization, and reasoning. In 2017, the World Wide Web Consortium (W3C) recommended an updated version of the SSN/SOSA Ontology [16] to be used when Semantic Web and Linked Data technologies are needed.

Work is pursuing to steer the IoT ecosystem towards the homogenization of the data to be transmitted, and tools to facilitate the integration of IoT devices into systems are being developed [8]. A similar problem, interoperability of multiple sensors from different manufacturers, is addressed in [4] by using SOSA/SSN for the development of semantics to manipulate this data. In [9], the M3 ontology was proposed as an extension of the SSN ontology, which allows reasoning on cross-domain $\mathrm{M} 2 \mathrm{M}$ sensor data using rules to infer contextual information. IoT-Lite [5] proposes a lightweight instantiation of the SSN ontology where the concepts Object, Service, Coverage are defined and where devices are divided into three groups Sensing Devices, Actuating Devices and Tag Devices but this is a different classification than the one we consider. In [2], a unified ontology (FIESTA-IoT) is presented with the aim of addressing interoperability issues, merging different IoT Ontologies (SSN, IoT-Lite, M3, among others).

If we consider domain-specific applications, we find that ontologies such as SAREF (Smart Appliance Reference) [6] that are close to ours. However, they do not manage a multi-level architecture and in this approach, devices are not related to each other.

Summarizing, we can observe that there is a need to develop new solutions to improve the interoperability of IoT devices. Generic solutions exist when there is a need to have a platform based on device observations but where the concern is simply to obtain sensor data, without having a more complex level of detail in the structure of the devices. When a higher granularity is introduced at the device level (by distinguishing, for instance, between gateways, nodes and sensors) no solutions have yet been found. This is the main objective of this work.

\section{Knowledge-Engineering Methodology}

We have been motivated by [14 to develop semantics that can be correct. As they suggest, there is no single way to develop semantics, and the modeling of a domain depends to some extent on several factors, including the purpose of the system it will support. The process of modeling an ontology is iterative. Below there is a summary of the steps to follow as proposed by [14]:

\section{Determine the domain and scope to be covered}

We have provided in the Introduction a description of the project domain and its intended use.

2. Sketch a list of competency questions that should be answered We give in Section 3.1 the competency questions that the ontology should be able to answer. 


\section{Consider reuse of existing ontologies}

In Section 2 there is an introduction to Semantic Web and ontologies related to the project domain. A detailed explanation is given in Section 5 .

4. Enumerate important terms in the ontology This is done in Section 4 when explaining our ontology.

\section{Develop the ontology}

The ontology we propose for connectivity management in IoT sensing is explained in Section 4 .

6. Validate the list of competency questions:

Competency questions are formulated in Section 3.1 and answered in 4 .

\subsection{Competency Questions}

One of the ways to determine the scope of an ontology is to make a list of questions, in order to obtain the baseline knowledge. These questions are called "Competency Questions". The answers of which should define the scope of the ontology. If the ontology is able to provide an answer for each question, it will mean that the described ontology contains enough information to work.

- Q1: Which elements or features can be observed by a sensor?

- Q2: How can an observation be made?

- Q3: How can the embedded sensor of a gateway be observed?

- Q4: How can the embedded sensor of a node be observed?

- Q5: How can an external sensor connected to a node be observed?

- Q6: If there are a batch of devices, is there any way to group them?

- Q7: Where is a given sensor located?

- Q8: How can we know which is the gateway providing an observation?

- Q9: Are there any other devices than gateway, node and sensor?

- Q10: How can one know if the device is enabled in a site?

These competency questions arise from the current industrial needs of Worldsensing and the IoT domain under consideration and are aimed at providing an abstract interpretation of the different implementations of the kind of IoT devices they handle, in order to be able to provide a uniform treatment of all this information. They are complete in the sense that they correspond to their actual needs, but this does not ensure that they will remain exactly as such forever.

\section{CMTS Ontology}

The Connectivity Management Tool Semantics (CMTS) Ontology is aimed at allowing tools for monitoring physical infrastructures through the use of three common types of IoT devices: gateways, nodes and sensors. The ontology builds from two key concepts: Site, i.e. a physical area being monitored, and the Devices installed in the Site to allow it. In our sites, three different types of devices co-exist: Gateways, Nodes, and Sensors. A Sensor may be either Hardware or 
Software and the structure of Gateways and Nodes can also be specified through the ontology. Finally, Observations are registered as a means to monitor the ObservableProperty of each Sensor.

The proposed ontology is depicted in Figure 3 by means of a UML Class Diagram that states the concepts of the domain that have to be specified in order to provide the aimed connectivity management in IoT sensing across different devices and settings of the sites being monitored. "cmts" is the default namespace when none is provided. A more detailed explanation of the concepts in the ontology is given in Section 4.2 .

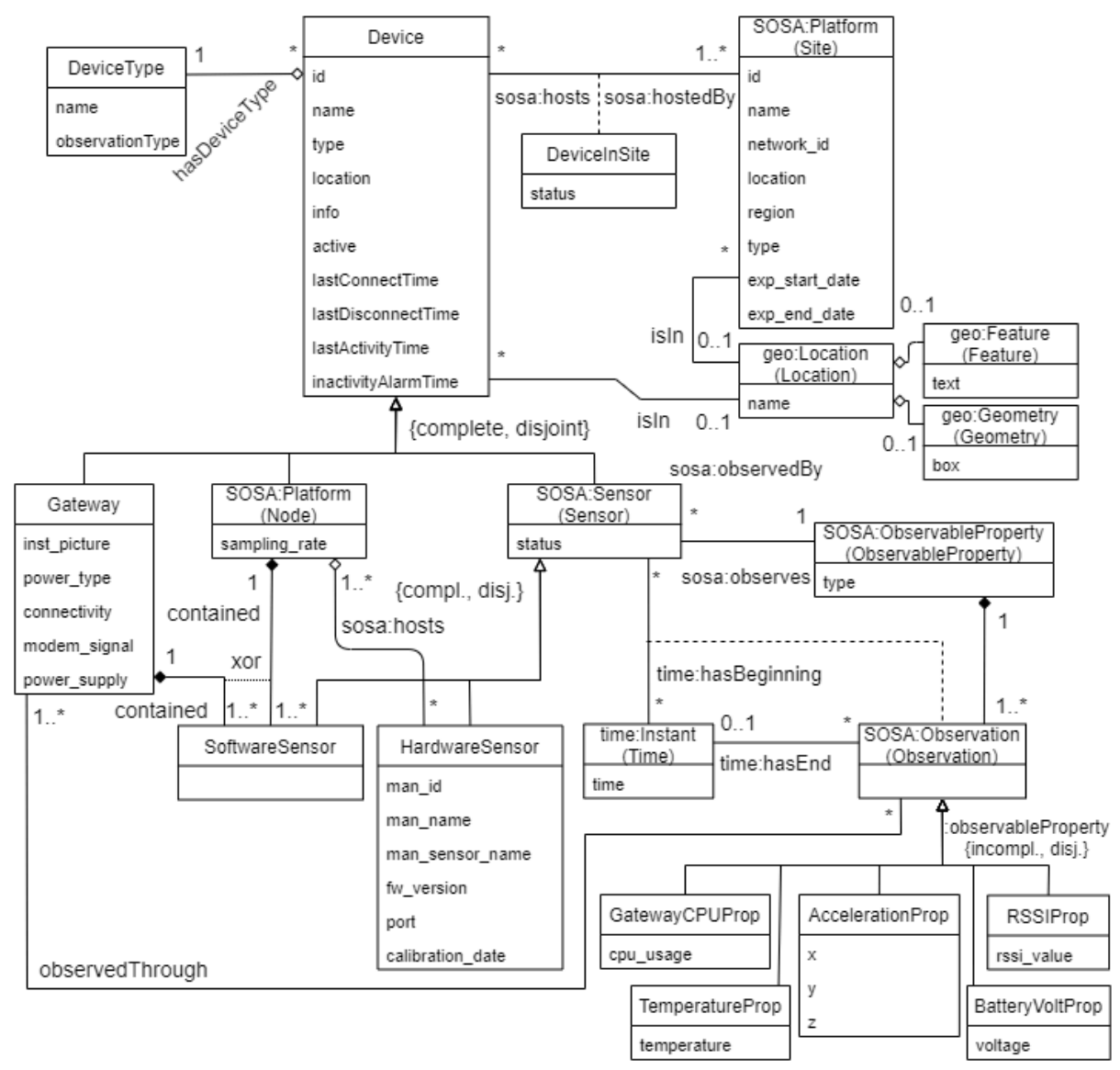

Fig. 3. CMTS Ontology

We have also had to specify some integrity constraints (i.e. conditions that any instance of the ontology should satisfy) to properly model the domain:

- C1: Location must have at least one Feature or one Geometry.

- C2: Sensors of a Node must be at the same Site as the Node. 
- C3: Sensors of a Gateway must be at the same Site as the Gateway.

- C4: Software Sensors must be at the same Location as the Gateway or Node they are connected to.

This ontology is complete in the sense that it incorporates all concepts and properties required by the IoT domain under consideration. Moreover, as it happens with all the approaches based on ontologies, it is easily extendable since new concepts and properties can be added to the ontology without having to modify the programs running on the current ontology.

\subsection{Resource URIs}

Using URIs increases the simplicity and manageability of systems. We show in Table 1 the main design of the URIs in our system. The first column represents the class name of the object, and the second represents the format of the URIs that have been generated. The BASE_URI refers to the URL entry point, located in a Worldsensing's domain. The format of the URIs follow the pattern:

\{BASE_URI\}:\{CLASS_NAME\}/\{CLASS_ID\}?\{CLASS_PROPERTY_1\}\&\{CLASS_PROPERTY_N\}

\begin{tabular}{|c|c|}
\hline Class & URI Patterns \\
\hline Site & cmts:Site/\{SiteName\}?\{LocationName\}\&\{Devices\} \\
\hline \multirow{2}{*}{ Gateway } & cmts:Device/\{DeviceName\}?\{DeviceType\}\&\{SoftwareSensors\} \\
\hline & $\&\{$ LocationName $\} \&\{$ SiteName $\}$ \\
\hline \multirow{2}{*}{ Node } & cmts:Device/\{DeviceName\}?\{DeviceType\}\&\{SoftwareSensors\} \\
\hline & $\&\{$ HardwareSensors $\} \&\{$ LocationName $\} \&\{$ SiteName $\}$ \\
\hline \multirow{2}{*}{ Hardware Sensor } & cmts:Device/\{DeviceName\}?\{NodeName\}\&\{DeviceType $\}$ \\
\hline & $\&\{$ ObservablePropertyName $\} \&\{$ LocationName $\} \&\{$ SiteName $\}$ \\
\hline \multirow{3}{*}{ Software Sensor } & cmts:Device/\{DeviceName\}?\{NodeName|GatewayName\} \\
\hline & $\&\{$ DeviceType $\&\{$ ObservablePropertyName $\&\{$ LocationName $\}$ \\
\hline & $\&\{$ SiteName $\}$ \\
\hline ObservableProperty & cmts: ObservableProperty/\{ObservablePropertyName\} \\
\hline \multirow{2}{*}{ Observation } & cmts: Observation/\{ObservablePropertyName\}?\{ObservationName\} \\
\hline & $\&\{$ Value $\} \&\{$ Time $\} \&\{$ SensorName $\} \&\{$ GatewayName $\}$ \\
\hline Location (Feature) & cmts:Location/\{LocationName\}?\{Feature\} \\
\hline Location (Geometry) & cmts:Location/\{LocationName\}?\{Geometry\} \\
\hline Time & cmts:Time/\{Date $\}$ T $\{$ Time $\}+\{$ TimeZone $\}$ \\
\hline
\end{tabular}

Table 1. Summary of the main URIs in CMTS

\subsection{Component Description}

Site A Site is a physical working area in the project. They are intended to group devices in the system by work areas so as to monitor these devices by granularity levels. It contains location information; the type of work being carried out; and, optionally, the start and end dates. This covers the competency question Q6.

Figure 4 provides a visual description of a Site having one Gateway, one Node, and one Sensor, whose Location is "LocationBCN". 


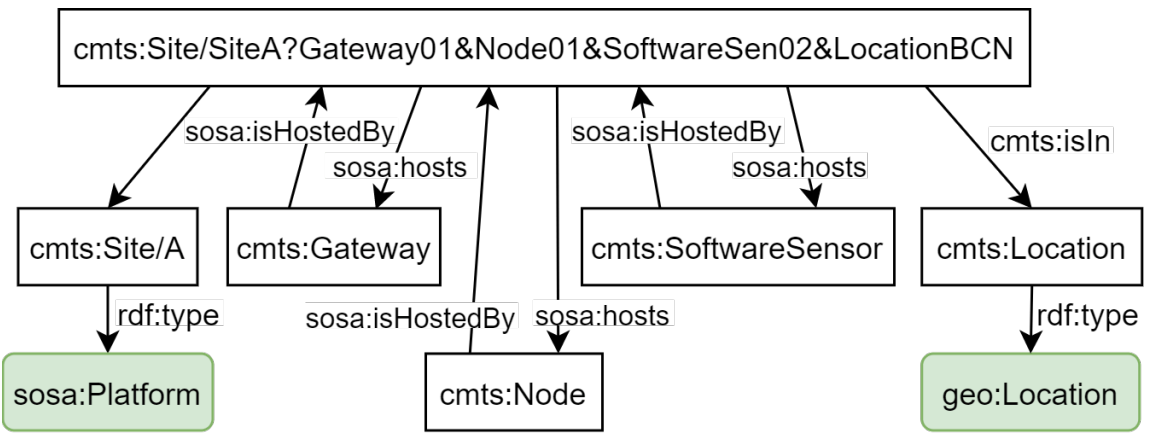

Fig. 4. Example of a Site description

Device Devices are the essential elements that carry out the monitoring of the infrastructures. A Site can contain multiple Devices. A Device is categorized by a DeviceType, such as the model name. This covers the competency question Q9. A Device is an element which can report to different Sites; the system can discern availability of a Device for each Site, whether it is active or not. This covers the competency question Q10. Devices have information about the infrastructure communication in terms of connection status and can handle Location information. Devices are classified in three types: Gateways, Nodes, and Sensors:

Gateway It is a physical element that acts as a router, gathering data from incoming wireless transmissions, grouping it and sending it to the data server, thus allowing to receive information sent wirelessly via the Nodes. Gateways are in charge of receiving the message via $L o R a$ and send it to the Internet. Gateways contain Sensors that capture metrics related to the Gateway such as the internal temperature or processor usage. For each Gateway we know its location, the way it communicates with the Sites, as well as other operational aspects.

Figure 5 provides a visual description of a Gateway having "GW_EU" as Device Type; contains a Software Sensor "SoftwareSen03" as Sensor; its Location is "LocationBCN", and it is in the Site "Site A".

Node It is a small physical element with limited processing capabilities, i.e. that of communicating directly to Sensors through a wired connection. They are responsible for collecting raw data measurements from the Sensors, building a well-formed message and sending it via $L o R a$ to the Gateways in range, and reporting Sensor failures. They also have internal Sensors to be monitored. The visual description of Node is similar to the one of Gateway given in Figure 5 .

Sensor They are the elements capable of making observations of a given feature. They can be either Software or Hardware. Software Sensors are used to monitor the infrastructure that supports the work, and we are only interested to know their sensor type, whether they work correctly and the values they report. They are the ones embedded into Gateways and Nodes and are used to 


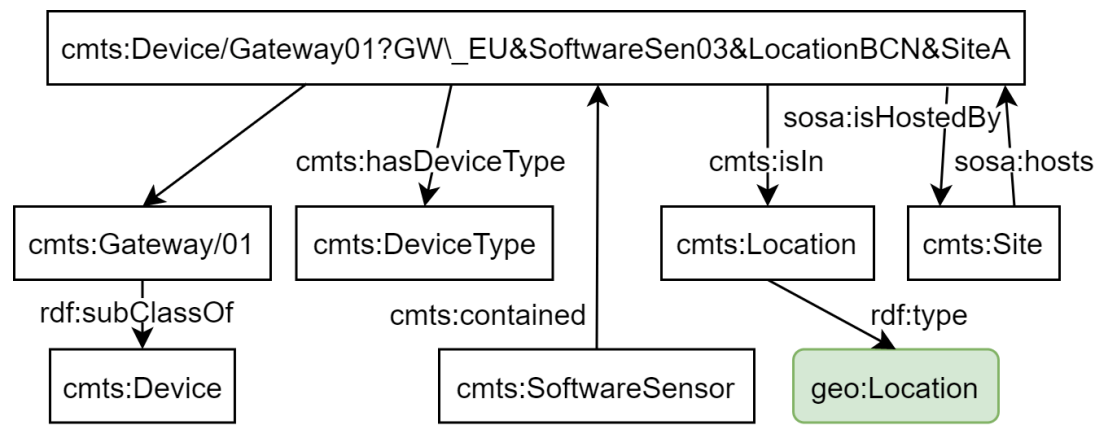

Fig. 5. Example of a Gateway description

monitor information like temperature, voltage, etc. This covers the competency questions Q3 and Q4. For Hardware Sensors we need to know data related to the manufacturer, the model, and specifics on how are they working and how they communicate. In addition to emphasizing the calibration of the data they report. They are the ones that are directly connected to the Nodes. This covers the competency question Q5.

Figure 6 provides a visual description about a Hardware Sensor. It has "Tiltmeter" as DeviceType; the Sensor has been named "HardwareSen01"; is connected to the Node "Node01"; is measuring ObservableProperty "Accelerometer"; its Location is "LocationBCN", and it is in the Site "Site A".

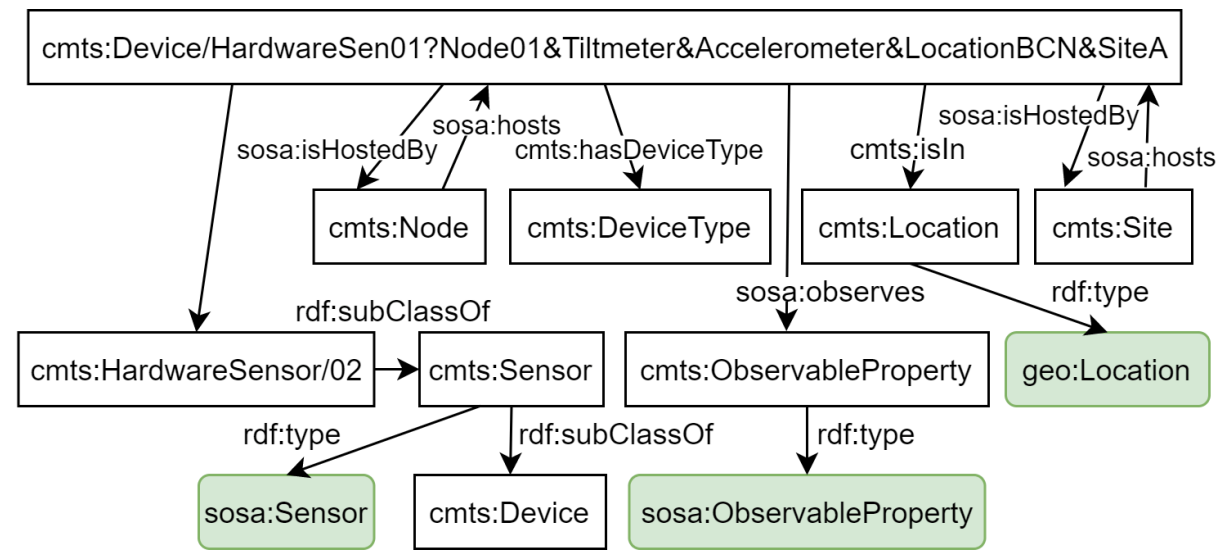

Fig. 6. Example of a Hardware Sensor description

ObservableProperty Are the properties to be observed in Sensors; for a given characteristic, property or feature. For example, the temperature inside a Gateway; the temperature of a sensor in an environment or the inclination of a surface. 
This covers the competency question Q1. Figure 3 explicitly distinguishes five possible ObservableProperty. However, other properties to be observed can be added when necessary as new subclasses of Observation.

Observation When a Sensor captures the value of an ObservableProperty at a given time it generates an Observation. In addition, it is possible to include a time interval to support those Observations that cannot obtain the value instantly. This covers the competency question Q2.

An Observation will always be generated from a Sensor. The Node is the device that sends it wirelessly as a radio signal. Gateways are the devices that will listen to this information, hence the "observedThrough" of an Observation, and will transmit it to the data server. This covers the competency question Q8.

Figure 7 and Figure 8 provide a visual description about an Observation made by a Hardware Sensor "HardwareSen01"; the communication of this $\mathrm{Ob}$ servation was made through the Gateway named "Gateway01"; is measuring ObservableProperty "Accelerometer"; its Location is "LocationBCN"; it is in the "Site A"; it also has the value measured " $0 \_0 \_9.8 \mathrm{~m} / 2$ ", and the Time has a value of "2021-03-15 at 16:15".

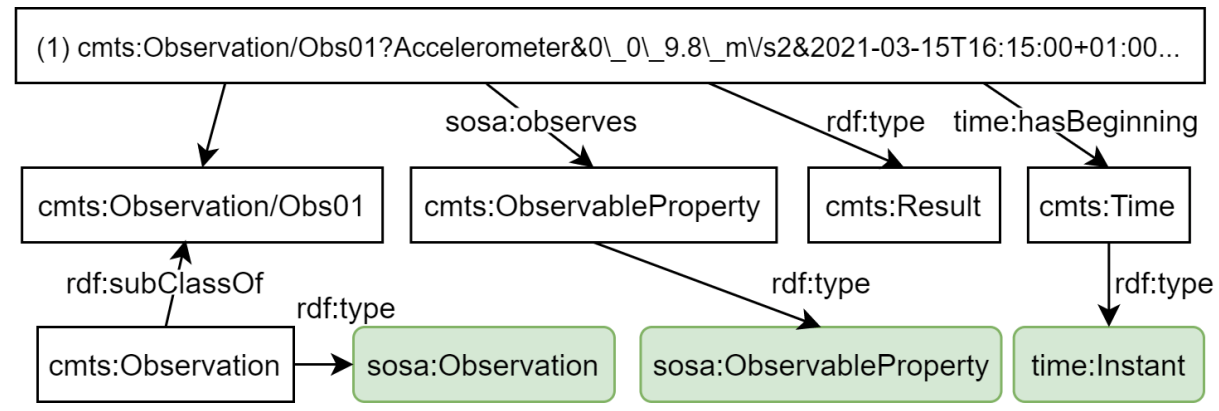

Fig. 7. Example of an Observation description - Part 1

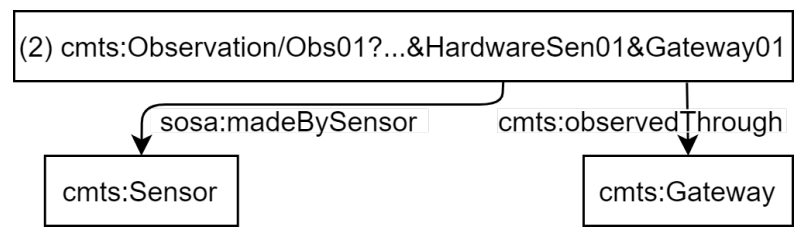

Fig. 8. Example of an Observation description - Part 2

Due to the use of LoRa communication technology; active communication between a Gateway and a Node is not possible. The Node sends out radio waves with the information, and the Gateways independently listen to these transmissions. More than one Gateway can receive the information. The server will only consider one packet as valid, and duplicated messages will be discarded. 
Location If desired, the user is given the possibility to indicate the location of the elements in the physical world. With this purpose, the system is provided with the ability of locating elements, as well as Sites and Devices, which comprise Gateways, Nodes and Sensors. This covers the competency question Q7.

\section{$5 \quad$ Matching Existing Ontologies}

The Semantic Sensor Network and Sensor Observation Sample Actuator (SSN/SOSA) ontology [16] is being used as the core ontology for the observation of measurements. Hence, a network of ontologies has been achieved, which is structured as follows (see Table 2 for the URLs):

- SSN/SOSA: Ontology to gather measurements from devices and sensors.

- GeoSPARQL: Ontology to describe geographical locations.

- OWL-Time: Ontology to describe temporal properties.

\begin{tabular}{ll} 
Prefix & Namespaces \\
\hline SSN & http://www.w3.org/ns/ssn/ \\
SOSA & http://www.w3.org/ns/sosa/ \\
GeoSPARQL http://www.opengis.net/ont/geosparql \\
OWL-Time & http://www.w3.org/2006/time
\end{tabular}

Table 2. Ontologies used in this project

In the following, we briefly explain how these ontologies have been used, along with the elements that have been newly added in order to define our solution.

\subsection{SSN/SOSA Ontology}

As said, SSN/SOSA is the ontology that comes closer to the ontology we propose here. This is why we use it as a baseline. As described in [16]: "SOSA provides a lightweight core for SSN and aims at broadening the target audience and application areas that can make use of Semantic Web ontologies. At the same time, SOSA acts as minimal interoperability fall-back level, i.e. it defines those common classes and properties for which data can be safely exchanged across all uses of SSN, its modules, and SOSA."

The SOSA/SSN classes that have been (re)used in our solution are:

- sosa:Platform, to describe:

- Sites, the physical working areas, which can contain multiple devices.

- Nodes, the physical elements that gather information from Sensors and send it to Gateways.

- sosa:Sensor, to describe the low-level Sensors that are collecting information.

- sosa:ObservableProperty, to specify the elements to be observed by a Sensor.

- sosa:Observation, to provide the value of the measurements of the ObservableProperty collected by the Sensors.

This is also true for some of the properties in our ontology that allow us giving more clarity to the actions being carried out: sosa:hostedBy, sosa:hosts, sosa:observedBy, sosa:observes. 


\subsection{OGC GeoSPARQL}

Several ontologies exist for the management of geospatial elements. However, we decided to use GeoSPARQL; an OGC Standard that has a small but powerful ontology that fits perfectly with the scope of this project. It enables describing data in Geographic Markup Language (GML) and also with Well-Known Text representation of geometry (WKT).

The GeoSPARQL classes that have been (re)used in our ontology are:

- geo:Location, to describe the location of Sites and Devices.

- geo:Feature, to specify points of interest from written language, in other words, as a String. For instance, "Barcelona".

- geo:Geometry, to define points of interest as coordinates from geometries such as points, lines and polygons, etc.

Moreover, we have used the properties geo:hasGeometry, geo:hasGML and geo:has WKT to complement the definition of geometries and spatial objects.

\subsection{OWL-Time Ontology}

The OWL-Time allows the Semantic Web to describe concepts and temporal properties. In our use case, this ontology is used to define when there has been an Observation, i.e. when a Sensor has taken a measurement. Using the properties time:hasBeginning and time:hasEnd. Also, through the class time:Instant we define the moment of time in which it has occurred.

\section{Conclusions and Future Work}

The interest in facilitating homogeneous information handling in IoT sensor networks has been acknowledged. Homogenization makes it simpler to enable compatibility between devices from different manufacturers. In this work, we proposed a semantics for the data management of gateways, nodes, and sensors, carrying out the theoretical and descriptive part of it. The ontology we used to specify such semantics builds upon recognized ontologies like SSN/SOSA or GeoSPARQL. We have used competency questions, stating what we expected from the semantics, to validate the appropriateness of our ontology.

As further work, we plan to incorporate additional features to our ontology to integrate IoT monitoring with the operational part of the company and we are also focused on the implementation of a system taking advantage of our ontology for IoT sensing of Worldsensing' sites.

As far as further applications, we have in mind the possibility of adding rule inference to the ontology, thus first enabling to develop applications with a context-awareness system and then allowing to incorporate an automatic notification system when a certain declarative condition over the data is met. 
Acknowledgements: This work is partially funded by Industrial Doctorates DI2019 from Generalitat de Catalunya and Grup de Recerca Consolidat IMP, 2017-SGR1749. Also with the support of inLab FIB at UPC, Worldsensing and the REMEDiAL project (TIN2017-87610-R). Also to the Worldsensing's team (T. Martinez-del-Hoyo and T. Fisher). We also thank the anonymous reviewers for their valuable comments.

\section{References}

1. Adelantado, F., Vilajosana, X., et al.: Understanding the Limits of LoRaWAN. IEEE Communications Magazine 55(9), 34-40 (2017)

2. Agarwal, R., Fernandez, D.G., et al.: Unified IoT ontology to enable interoperability and federation of testbeds. In: IEEE World Forum on Internet of Things (WF-IoT). pp. 70-75 (2016)

3. Allemang, D., Hendler, J.: Semantic Web for the Working Ontologist. Morgan Kaufmann, 1st edn. (2008)

4. Berges, I., Ramírez-Durán, V.J., et al.: Facilitating Data Exploration in Industry 4.0. In: Advances in Conceptual Modeling. International Conference on Conceptual Modeling. ER 2020. pp. 125-134 (2020)

5. Bermúdez-Edo, M., Elsaleh, T., et al.: IoT-Lite Ontology (2015), https://www.w3. org/Submission/iot-lite/, [Last accessed 25 Mar 2021]

6. Daniele, L., den Hartog, F., et al.: Study on semantic assets for smart appliances interoperability. Tech. rep., TNO (2015)

7. Eid, M., Liscano, R., et al.: A universal ontology for sensor networks data. In: IEEE International Conference on Computational Intelligence for Measurement Systems and Applications. CIVEMSA 2007. pp. 59-62 (2007)

8. Frigo, M., Hirmer, P., et al.: A Toolbox for the Internet of Things - Easing the Setup of IoT Applications. In: ER Forum, Demo and Posters. International Conference on Conceptual Modeling. ER 2020. pp. 80-100 (2020)

9. Gyrard, A., Bonnet, C., et al.: Enrich machine-to-machine data with semantic web technologies for cross-domain applications. In: IEEE World Forum on Internet of Things (WF-IoT). pp. 559-564 (2014)

10. Haller, A., Janowicz, K., et al.: The modular ssn ontology: A joint w3c and ogc standard specifying the semantics of sensors, observations, sampling, and actuation. Semantic Web Journal (2018)

11. IoT Analytics: Why the Internet of Things is called Internet of Things: Definition, history, disambiguation (2014), https://iot-analytics.com/internet-of-thingsdefinition, [Last accessed 25 Mar 2021]

12. Janowicz, K., Haller, A., et al.: Sosa: A lightweight ontology for sensors, observations, samples, and actuators. Journal of Web Semantics 56, 1-10 (2019)

13. Mekki, K., Bajic, E., et al.: A comparative study of lpwan technologies for largescale iot deployment. ICT Express pp. 1-7 (2019)

14. Noy, N.F., McGuiness, D.L.: Ontology development 101: A guide to creating your first ontology. Tech. rep., Knowledge Systems - Stanford University (2001)

15. Vila, M., Sancho, M.R., Teniente, E.: XYZ Monitor: IoT Monitoring of Infrastructures using Microservices. In: Service-Oriented Computing - ICSOC 2020 Workshops. pp. 472-484 (2021)

16. W3C - OGC: Semantic Sensor Network Ontology - W3C Recommendation (2017), https://www.w3.org/TR/vocab-ssn/, [Last accessed 18 Apr 2021]

17. W3C Incubator Group: Semantic Sensor Network XG Final Report (2011), https: //www.w3.org/2005/Incubator/ssn/XGR-ssn/, [Last accessed 23 Apr 2021] 